Pacific Journal of Mathematics

THE EMBEDDING OF HOMEOMORPHISMS OF THE PLANE
IN CONTINUOUS FLOWS 


\title{
THE EMBEDDING OF HOMEOMORPHISMS OF THE PLANE IN CONTINUOUS FLOWS
}

\author{
GARY D. JONES
}

\begin{abstract}
A study of fundamental regions of the plane under an orientation preserving, fixed point free, self-homeomorphism of the plane is made under the conditions that there are finitely many fundamental regions $R_{i}$ under $f$, if $x \in R_{i}$ Int $R_{i}$, then $x \in C \subset R_{i}$ - Int $R_{i}$ where $C$ is a proper flowline, and if $x_{1}$ and $x_{2}$ are in Int $R_{i}$, then $x_{1} \sim x_{2} \bmod \operatorname{Int} R_{i}$. The topological structure of the fundamental regions is determined. Using these results, it is shown that in certain cases the embedding problem can be reduced to a problem of extending a continuous flow defined on an open set to the closure of the set.

In the last section, sufficient conditions for self-homeomorphisms of the plane and the closed unit disc with one fixed point to be embedded in continuous flows are given.
\end{abstract}

Let $X$ be a topological space and let $G$ denote a topological group. The ordered triple $(X, G, \pi)$ is a dynamical system if (1) $\pi: X \times G \rightarrow X$ is continuous, (2) $\pi\left(\pi\left(x, g_{1}\right), g_{2}\right)=\pi\left(x, g_{1}+g_{2}\right)$, and $(3) \pi(x, e)=x$ for every $x \in X$. If $G$ is the additive group of real numbers, then ( $X$, $G, \pi)$ is called a continuous flow. If $G$ is the additive group of integers, then $(X, G, \pi)$ is a discrete flow.

This paper is concerned with the following problem. If $G$ is a given topological group and $\left(X, G^{*}, \pi^{*}\right)$ is a given dynamical system, where $G^{*}$ is a subgroup of $G$ with the relative topology, then find a dynamical system $(Y, G, \pi)$ such that $(1) \pi\left(y, g^{*}\right)$ for $g^{*} \in G^{*}$ is invariant on a subset $Z$ of $Y$, (2) $Z$ is homeomorphic to $X$, and (3) $\pi$ on $Z \times G^{*}$ is topologically equivalent to $\pi^{*}$ on $X \times G^{*}$, i.e. there is a homeomorphism $h: X \rightarrow Z$ such that $h^{-1}\left(\pi\left(h(x), g^{*}\right)\right)=\pi^{*}\left(x, g^{*}\right)$. If $G$ is the additive group of real numbers and $G^{*}$ is the additive group of integers, then the problem is that of embedding a selfhomeomorphism in a continous flow.

If the space $X$ is allowed to be enlarged in order to accommodate $\pi$, the problem is referred to as the unrestricted problem. If $X=$ $Y=Z$ the problem is referred to as the restricted problem [5].

If $G$ is the additive group of real numbers and $G^{*}$ is the integers, then the unrestricted problem is easily solved [5], [7], [8] for any topological space $X$ and any self-homeomorphism.

The restricted problem is only partially solved. Fine and Schweigert [2] and Fort [6] proved that a self-homeomorphism $T$ of an interval can be embedded in a continuous flow if and only if it is order pre- 
serving. Utz [11] gave a constructive proof of this theorem.

Foland and Utz [5] solved the restricted problem where $T$ is an orientation preserving self-homeomorhism of a circle. Foland [3] has shown that if $T$ is an almost periodic, orientation preserving selfhomeomorphism of a closed 2-cell, then $T$ can be embedded in a continuous flow on the 2-cell. Moreover, if $T$ is almost periodic but not periodic, then the embedding is unique.

Andrea [1] has given sufficient conditions for a fixed point free self-homeomorphism of the Euclidean plane to be embedded in a continuous flow.

2. Fundamental regions of the plane. In this section numerous properties of fundamental regions of the plane will be given, and using these properties it will be possible to give a description of the plane when it consists of $n$ fundamental regions.

In this section $f$ will be a self-homeomorphism of the plane which is orientation preserving and which has no fixed points.

Definition 1. [1]. If $p$ and $q$ are points in the plane, then $p \sim q$ if $p$ and $q$ are endpoints of some curve segment $C$ (the homeomorph of the closed unit interval) for which $f^{n}(C) \rightarrow \infty$ as $n \rightarrow \pm \infty$.

THEOREM 1. [1] The relation $\sim$ is an equivalence relation.

Definition 2. [1] The fundamental regions of the plane under $f$ are the equivalence classes of the equivalence relation $\sim$.

Definition 3. If $A$ is a subset of the plane, then $x \sim y \bmod A$ if $x$ and $y$ are endpoints of some curve segment $C \subset A$ such that for every compact set $K$ in the plane there is a natural number $N$ such that $f^{n}(C) \cap K=\phi$ when $|n|>N$.

Definition 4. [1]. A proper flowline for $f$ is a subset $F$ of the plane such that

(a) $f(F)=F$,

(b) $F$ is a one-to-one continuous image of the real line, and

(c) $F \cup\{\infty\}$ is a Jordan curve on the sphere $R^{2} \cup\{\infty\}$.

The following three conditions will be assumed in this section and in $\S 3$.

(1) There exists exactly $n$ fundamental regions $R_{i}$ under $f$.

(2) If $x \in R_{i}-$ Int $R_{i}$, then $x \in C \subset R_{i}$ - Int $R_{i}$ where $C$ is a proper flowline.

(3) If $x_{1}, x_{2} \in \operatorname{Int} R_{i}$, then $x_{1} \sim x_{2} \bmod$ Int $R_{i}$.

We will let $R$ or $R$ with a subscript denote fundamental regions of 
the plane under $f$.

It follows from the following remark that each fundamental region is $f$-invariant.

REMARK 1. [1]. Suppose that $\left\{E_{i}\right\}$ is a finite collection of disjoint arcwise connected sets. If each $f\left(E_{i}\right)=E_{j}$, then $f\left(E_{i}\right)=E_{i}$ for every $i$.

\section{REMARK 2.}

(a) $R$ is arcwise connected,

(b) $R$ is unbounded, and

(c) if $C$ is a simple closed curve contained in $R$, then Int $(C) \subset R$.

Proof. (a) and (b) follow directly from the definitions. Suppose that (c) does not hold. Then there is $x \in \operatorname{Int} C$ such that $x \notin R$. Suppose $x \in S$ where $S$ is a fundamental region under $f$. Since $f^{n}(x) \rightarrow$ $\infty$, as $n \rightarrow \infty, f^{n}(x) \notin C \cup \operatorname{Int} C$ for some $n$. But $f^{n}(x) \in S$ and $S$ is arc wise connected. Therefore, there is an arc $L$ joining $x$ and $f^{n}(x)$ where $L \subset S$. But that is not possible since $L \cap C \neq \phi$.

REMaRK 3. If there exists $x \in R_{i}-\operatorname{Int} R_{i}$, then $x \in C$, where $C$ is defined by (2), and $C$ divides the plane into two open half planes $H_{1}$ and $H_{2}$ such that

(a) $x_{1}, x_{2} \in$ Int $R_{i}$ implies $x_{1}$ and $x_{2}$ are in the same half plane,

(b) if Int $R_{i} \neq \phi$ and if $x \in C$, then there is a neighborhood $U$ of $x$ such that $U \cap H_{1} \subset \operatorname{Int} R_{i}$ where Int $R_{i} \subset H_{1}$, and (c) if Int $R_{i}=\phi$, then $R_{i}=C$.

Proof. (a) Let $x_{1}$ and $x_{2} \in \operatorname{Int} R_{i}$. Let $x_{1} \in H_{1}$ and $x_{2} \in H_{2}$. By (3), there is an arc $A$ joining $x_{1}$ and $x_{2}$ such that $A \subset \operatorname{Int} R_{i}$. Therefore, $A \cap C \neq \phi$, which is impossible since $C \subset R_{i}-\operatorname{Int} R_{i}$.

(b) Let $x \in C$. Let $x_{1} \in \operatorname{Int} R_{i}$. Then there is a curve $L \subset R_{i}$ such that $x_{1}$ and $y$ are endpoints of $L$, where $y \in C$, and $L \cap C=\{y\}$. Since $f(C)=C$ and $f$ is fixed point free, there are integers $m$ and $n$ such that $f^{n}(y)$ and $f^{m}(y)$ are separated on $C$ by $x$.

If $f^{n}(L) \cap f^{m}(L) \neq \phi$, let $L_{1}$ be the smallest closed subsegment of $f^{n}(L)$ that contains $f^{n}(y)$ such that $L_{1} \cap f^{m}(L) \neq \phi$. Let $L_{2}$ be the smallest closed subsegment of $f^{m}(L)$ that contains $f^{m}(y)$ such that $L_{2} \cap L_{1} \neq \phi$. If $C_{1}$ is the closed subsegment of $C$ from $f^{m}(y)$ to $f^{n}(y)$, then $C_{1} \cup L_{1} \cup L_{2}$ forms a simple closed curve. Let $\varepsilon=\min \left\{d\left(x, L_{1}\right)\right.$, $\left.d\left(x, L_{2}\right)\right\}$. Then if $S=\{z: d(z, x)<\varepsilon\}, S \cap H_{1}$ is contained in the simple closed curve $C_{1} \cup L_{1} \cup L_{2}$, which, by Remark 2, is contained in $R_{i}$.

There is an arc $K \subset \operatorname{Int} R_{i}$ such that $f^{n}\left(x_{1}\right)$ and $f^{m}\left(x_{1}\right)$ are its endpoints. If $f^{n}(L) \cap f^{m}(L)=\phi$, let $L_{1}$ be the smallest closed subsegment of $f^{n}(L)$ containing $f^{n}(y)$ such that $L_{1} \cap K=\left\{z_{1}\right\}$. Let $L_{2}$ be the 
smallest closed subsegment of $f^{m}(L)$ containing $f^{m}(y)$ such that $L_{2} \cap$ $K=\left\{z_{2}\right\}$. Let $K_{1}$ be the subsegment of $K$ such that $z_{1}$ and $z_{2}$ are its endpoints. Now $C_{1} \cup K_{1} \cup L_{1} \cup L_{2}$ is a simple closed curve. Let $S=$ $\{u: d(x, u)<\varepsilon\}$ where $\varepsilon=\min \left\{d\left(x, K_{1}\right), d\left(x, L_{1}\right), d\left(x, L_{2}\right)\right\}$. Then $S \cap H_{1}$ is contained in the simple closed curve $C_{1} \cup L_{1} \cup L_{2} \cup K_{1}$ which is contained in $R_{i}$.

(c) If $x \in R_{i}$, then by (2) there is a proper flowline $C$ such that $x \in C$. If $y \in R_{i}-C$ then there is a proper flowline $C^{\prime}$ such that $y \in C^{\prime}$.

If $C \cap C^{\prime} \neq \phi$, let $L_{1}^{+}$be the closed half line from $y$ containing $f(y)$ and $L_{1}^{-}$the closed half line from $y$ containing $f^{-1}(y)$. Let $\mathrm{C}_{1}$ and $C_{2}$ be the smallest closed subsegments of $L_{1}^{-}$and $L_{1}^{+}$respectively such that $C_{i} \cap C=\left\{x_{i}\right\}$ for $i=1,2$. Such segments do exist because $C^{\prime} \cap$ $C \neq \phi, y \notin C$, and the properties of $f$. Thus the segment from $x_{1}$ to $x_{2}$ on $C, C_{1}$ and $C_{2}$ form a simple closed curve and every point in its interior must be in $R_{i}$, which is not possible.

If $C \cap C^{\prime}=\phi$, we can join $C$ to $C^{\prime}$ by a segment $A \subset R_{i}$ such that $A \cap C=\left\{x_{1}\right\}$ and $A \cap C^{\prime}=\left\{x_{2}\right\}$. Let $C_{i}$ be the closed subsegments of $C$ and $C^{\prime}$ respectively from $x_{i}$ to $f\left(x_{i}\right)$ for $i=1,2$. Let $A_{1}$ be the smallest closed subsegment of $f(A)$ such that $A_{1} \cap\left(A \cup C_{2}\right) \neq \phi$. Now $C_{1}, A_{1}$, and $\left(A \cup C_{2}\right)$ from $x_{1}$ to $A_{1} \cap\left(A \cup C_{2}\right)$ is a simple closed curve and the result follows.

REMARK 4. If $C_{1}$ and $C_{2}$ contained in $R_{i}$ are curves defined as in (2) such that $C_{1} \neq C_{2}$, then $C_{1} \cap C_{2}=\phi$. Also, if $C_{1}$ is a curve defined as in (2), then $R_{i}$ is contained in the closed half plane defined by $C_{1}$ containing Int $R_{i}$.

Proof. Suppose $C_{1} \neq C_{2}$, but $C_{1} \cap C_{2} \neq \phi$. Suppose $y \in C_{1}-C_{2}$. Let $A$ be a closed subsegment of $C_{1}$ containing $y$ such that $x_{1}$ and $x_{2}$ are endpoints of $A, x_{1}$ and $x_{2} \in C_{1} \cap C_{2^{\prime}}$ and $\left(A-\left\{x_{1}, x_{2}\right\}\right) \cap C_{2}=\phi$ for $i=1,2$. Now $A$ together with the subsegment of $C_{2}$ from $x_{1}$ to $x_{2}$ forms a simple closed curve $K$. Let $z \in$ the interior of $K$. Now $f^{n}(z) \rightarrow \infty$ as $n \rightarrow \infty$, thus $f^{n}(z) \in$ the interior of $f^{n}(K)$ but $f^{n}(z) \notin$ interior $K$ for some integer $n$. Now $z$ and $f^{n}(z) \in \operatorname{Int} R_{i}$ by Remark 2 , but $z$ and $f^{n}(z)$ can not be connected by an arc in Int $R_{i}$ which is contrary to (3).

Suppose there is $x \in R_{i}$ but in the opposite open half plane, determined by $C_{1}$, from Int $R_{i}$. Then by the above paragraph and (2), there is a proper flowline $C_{2}$ such that $x \in C_{2}$ and $C_{1} \cap C_{2}=\phi$. Now the proof of Remark $3 \mathrm{c}$ can be applied to show that there must be a point in the same half plane as $x$ which is in Int $R_{i}$, which is not possible.

THEOREM 2. [1]. The self-homeomorphism $f$ is equivalent to a 
translation if and only if it has exactly one fundamental region.

REMARK 5. Let $C$ be a proper flowline of $f$. Then there is a homeomorphism $g$ such that $\left.g\right|_{c}=\left.f\right|_{c}$ and such that $g$ is topologically equivalent to a translation.

Proof. Since $C$ is a proper flowline of $f$ there is a selfhomeomorphism $h$ of the plane where $h(C)=L$ and where $L$ is the $y$-axis. Define $T: P \rightarrow P$, where $P$ is the plane, by

$$
\begin{aligned}
& T(0, y)=h f h^{-1}(0, y), \text { where }(0, y) \in L \\
& T(x, y)=\left(x,\left(h f h^{-1}(0, y)\right)_{y}\right) \text { where }(x, y) \notin L
\end{aligned}
$$

and where $\left(h f h^{-1}(0, y)\right)_{y}$ denotes the $y$ coordinate of $h f h^{-1}(0, y)$. Then $T$ is an orientation preserving self-homeomorphism of the plane with no fixed points and with exactly one fundamental region. Therefore, $g=h^{-1} T h$ has this property, $\left.g\right|_{c}=\left.f\right|_{c}$, and $g$ is equivalent to a translation by Theorem 2 .

REMARK 6. If $R$ - Int $R$ contains two distinct proper flow lines $C_{1}$ and $C_{2}$, then $R$ is the closed strip bounded by $C_{1}$ and $C_{2}$.

Proof. By Remark 3, if $C_{1}$ and $C_{2}$ are distinct proper flow lines in $R$-Int $R$, then Int $R \neq \phi$. Let $H_{j}^{1}$ and $H_{j}^{2}$ be the two closed half planes defined by $C_{j}$. By Remark 4 , it can be assumed that $R \subset H_{1}^{2}$. Therefore, $C_{2} \subset H_{1}^{2}-C_{1}$. Let $H_{2}^{1}$ be the half plane containing $H_{1}^{1}$. Then, $R \subset H_{2}^{1}$ and therefore $R \subset H_{2}^{1} \cap H_{1}^{2}$. Let $x_{3} \in H_{2}^{1} \cap H_{1}^{2}-\left(C_{1} \cup C_{2}\right)$. Let $B$ be a closed segment joining $C_{1}$ and $C_{2}$ such that $x_{3} \in B, B \cap C_{1}=$ $\{y\}$, and $B \cap C_{2}=\{z\}$. Since $y \sim z$, there is an arc $A \subset R$ joining $y$ and $z$ such that $f^{n}(A) \rightarrow \infty$ as $n \rightarrow \pm \infty$. Let $A_{1}$ be a closed subsegment of $A$ such that $A_{1} \cap C_{1}=\left\{y_{1}\right\}$ and $A_{1} \cap C_{2}=\left\{z_{1}\right\}$. There is an integer $N>0$ such that if $n>N, f^{n}\left(y_{1}\right)$ and $f^{-n}\left(y_{1}\right)$ are separated on $C_{1}$ by $y$, and $f^{n}\left(z_{1}\right)$ and $f^{-n}\left(z_{1}\right)$ are separated on $C_{2}$ by $z$. Since $f^{n}\left(A_{1}\right) \rightarrow$ $\infty$ as $n \rightarrow \pm \infty$, there is an $M>N$ such that $f^{M}\left(A_{1}\right), f^{-M}\left(A_{1}\right)$, and the closed subsegments of $C_{1}$ and $C_{2}$ from $f^{M}\left(y_{1}\right)$ to $f^{-M}\left(y_{1}\right)$ and $f^{M}\left(z_{1}\right)$ to $f^{-M I}\left(z_{1}\right)$ respectively form a simple closed curve which contains $x_{3}$ in its interior. But that implies $x_{3} \in \operatorname{Int} R$.

REMaRK 7. If $x \in\left(R_{i}-\operatorname{Int} R_{i}\right) \cap \bar{R}_{k}$, then there is an arc $A$ joining a point of $R_{k}$ to $x$, where $A-\{x\} \subset R_{k}$. Further, if $x \in C$, where $C$ is defined as in (2), then for every $w \in C$, there is a neighborhood $U$ of $w$ such that $U \cap\left(H^{\prime}-C\right) \subset R_{k}$, where $H^{\prime}$ is the closed half plane defined by $C$ which contains $R_{k}$. 
Proof. Let $x \in C_{i}$, where $C_{i}$ is defined by (2). Let the closed half planes defined by $C_{i}$ be $H_{i}^{1}$ and $H_{i}^{2}$. Suppose $R_{k} \subset H_{i}^{2}$. By Remark 6 , there are at most $2 n$ distinct curves $C_{j}$ defined as in (2). Let $\alpha=$ $\min _{C_{j} \subset\left(H_{i}^{2}-C_{i}\right)}\left\{d\left(x, C_{j}\right)\right\}$. Consider the open disc $S$ with center at $x$ and radius $\alpha / 2$. Since $x \in \bar{R}_{k}$, there exists $y \in R_{k} \cap S$. Suppose $z \epsilon$ $S \cap\left(H_{i}^{2}-C_{i}\right)$, where $z \notin R_{k}$. Since $\left(\cup_{j} R_{j}\right) \cap S=S$, there exists $z_{0} \in$ $S \cap\left(H_{i}^{2}-C_{i}\right)$ where $z_{0} \in R_{k_{0}}$ - Int $R_{k_{0}}$. But then there would exist a curve $C_{k_{0}}$ defined by (2), where $C_{k_{0}} \cap S \cap\left(H_{i}^{2}-C_{i}\right) \neq \phi$, which is not possible. The proof of the last part is similar to the proof of Remark $3 \mathrm{~b}$.

REMARK 8. If $C$ is an arcwise connected component of $\bar{R}-\operatorname{Int} R$, then $C$ is a proper flow line contained in exactly one fundamental region.

Proof. Let $x \in C$. Then $x \in\left(R_{j}-\operatorname{Int} R_{j}\right) \cap \bar{R}_{k}$ for some fundamental regions $R_{j}$ and $R_{k}$. If Int $R_{j} \neq \phi$, by Remarks 3 and 7, there is a neighborhood $U$ of $x$ such that $U \subset R_{j} \cup R_{k}$. Thus, $R$ is $R_{j}$ or $R_{k}$. Let $x \in C_{1}$, where $C_{1}$ is given by (2). Remarks 3 and 7 imply $C_{1} \subset C$. If $w \in C-C_{1}$, there must be an arc in $\bar{R}-\operatorname{Int} R$ from $w$ to $C_{1}$, which is not possible by Remarks 3 and 7. If Int $R_{j}=\phi$ and if $R_{j}=R$ the result follows from Remark 3. Otherwise if $x \in C$, then $x \in R_{j} \cap \bar{R}$. Now applying Remark 7, it follows that $C_{1}=C$.

Theorem 3. [1] The plane under $f$ can not have exactly two fundamental regions.

The notation $\#(R)$ will be used to represent the number of arcwise connected components of $\bar{R}-$ Int $R$.

REMARK 9. If $\sharp(R)=1$, then $R$ is a proper flow line or a closed half plane.

Proof. If Int $R=\phi$, then $R$ is a proper line by Remark 2. If Int $R \neq \phi$, then by Remarks 3,7 and $8, R$ is an open half plane or a closed half plane.

Suppose $R$ is not closed. Then $\bar{R}-$ Int $R \subset R_{2}$. Thus, there exists $x \in\left(R_{2}-\operatorname{Int} R_{2}\right) \cap \bar{R}$. Let $x \in C$, where $C$ is defined as in (2). Let $H_{1}^{2}$ and $H_{1}^{1}$ be the closed half planes defined by $C$. By Remark 2, it can be assumed that $R \subset H_{1}^{1}$, and then, by Remarks 3 and $4 R_{2} \subset$ $H_{1}^{2}$. Using Remark 5, define $g$ such that $\left.g\right|_{c}=\left.f\right|_{c}$. Define $F: P \rightarrow$ $P$, where $P$ is the plane, by

$$
F(x)= \begin{cases}f(x) & \text { if } x \in H_{1}^{1} \\ g(x) & \text { if } x \in H_{1}^{2}\end{cases}
$$

Then $F$ is an orientation preserving self-homeomorphism of the plane with no fixed points, and the plane under $F$ has exactly one funda- 
mental region, since, by Theorem 3 , it can not have two fundamental regions. Let $A$ be any arc joining $y \in R$ to $x$, where $A \subset H_{1}^{1}$. Then $f^{n}(A)=F^{n}(A) \rightarrow \infty$ as $n \rightarrow \pm \infty$. Therefore, $x \in R$, and it follows that $R$ is closed.

REMARK 10. If $\sharp(R)=2$, then $R$ is either an open strip or a closed strip.

Proof. Let $C_{1}$ and $C_{2}$ be the two arcwise connected components of $\bar{R}$ - Int $R$. It follows that the interior of the strip bounded by $C_{1}$ and $C_{2}$ is contained in $R$, and that $R$ is contained in the closed strip bounded by $C_{1}$ and $C_{2}$.

Suppose $x_{1} \in R-$ Int $R$. Then $x_{1} \in C_{1}$ or $x_{1} \in C_{2}$. Suppose $x_{1} \in C_{1}$. By Remark 8, $C_{1} \subset R$.

Using Remark 5, define $g_{i}$ where $g_{i}$ agrees with $f$ on $C_{i}, i=1,2$. Define $F: P \rightarrow P$, where $P$ is the plane, by

$$
F(x)= \begin{cases}g_{1}(x) & \text { if } x \in \text { closed half plane defined by } C_{1} \text { not con- } \\ & \text { taining Int } R \\ f(x) & \text { if } x \in \text { closed strip bounded by } C_{1} \text { and } C_{2} \\ g_{2}(x) & \text { if } x \in \text { closed half plane defined by } C_{2} \text { not con- } \\ & \text { taining Int } R .\end{cases}
$$

Then $F$ is an orientation preserving self-homeomorphism of the plane, and the plane has at most two fundamental regions under $F$. Therefore, by Theorem 3, it has exactly one. It now follows that $C_{2} \subset R$.

REMARK 11. If $\sharp(R) \geqq 3$, then either $R$ is open or else $R$ contains just one of the components of $\bar{R}-\operatorname{Int} R$.

Proof. If $R$ contains at least two of the components of $\bar{R}-\operatorname{Int} R$, then by Remark $6, \#(R)=2$.

Consider the homeomorphism defined in Figure 1. Each point $x$ lies on a curve. Let $f(x)$ be in the direction of the arrow along the curve one unit. The homeomorphism $f$ satisfies (1), (2), and (3), and $\#\left(R_{4}\right)=3$ but $R_{4}$ is not open.

REMARK 12. If $R_{1}$ and $R_{2}$ are not separated and $R_{2}$ and $R_{3}$ are not separated, then $R_{1}$ and $R_{3}$ are separated by $R_{2}$.

Proof. Since $R_{1}$ and $R_{2}$ are not separated, either there exists $x_{2} \epsilon$ $\left(R_{2}-\right.$ Int $\left.R_{2}\right) \cap \bar{R}_{1}$, or there exists $x_{2} \in\left(R_{1}-\right.$ Int $\left.R_{1}\right) \cap \bar{R}_{2}$.

Case I. Suppose $x_{2} \in\left(R_{2}\right.$ - Int $\left.R_{2}\right) \cap \bar{R}_{1}$. Let $x_{2} \in C_{2}$, where $C_{2}$ is defined by (2). Let $H_{2}^{1}$ and $H_{2}^{2}$ be the closed half planes defined by 

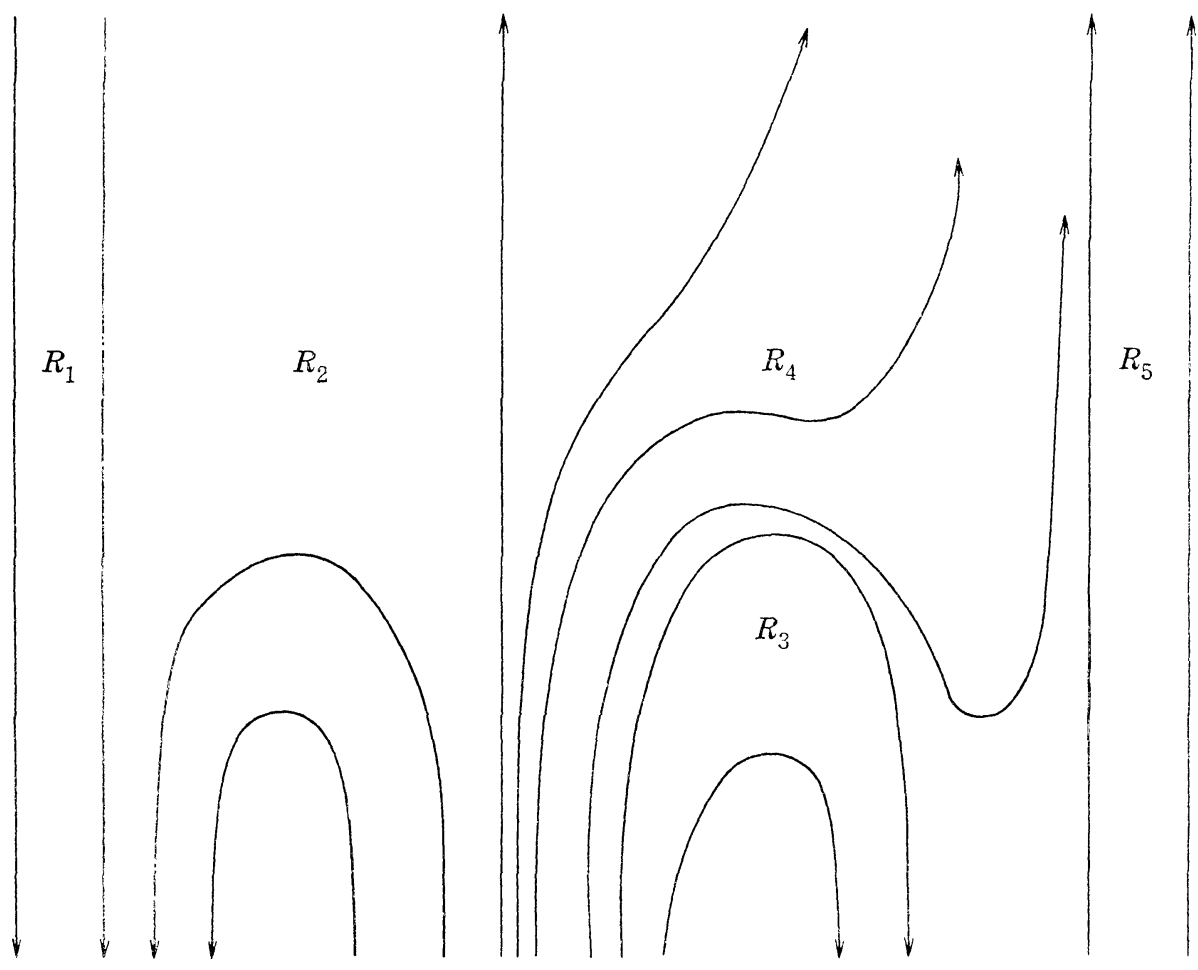

FIGURE 1

$C_{2}$. Suppose for definiteness that $R_{1} \subset H_{2}^{1}-C_{2}$ and then by Remark $3, R_{2} \subset H_{2}^{2}$. By Remark 7, for every $w \in C_{2}$, there is a neighborhood $U$ of $w$ such that $U \cap\left(H_{2}^{1}-C_{2}\right) \subset R_{1}$. Since $R_{3}$ and $R_{2}$ are not separated, $R_{3} \subset H_{2}^{2}-C_{2}$. Therefore, $R_{1}$ and $R_{3}$ are separated by $R_{2}$.

Case II. The proof is similar to Case I.

Theorem 4. Suppose $R_{i}$ and $R_{i+1}$ are not separated for $i=1,2$, $\cdots, n-1$. Then
(a) $R_{i}$ and $R_{k}$ are separated for $k \neq i-1, i+1$,
(b) $R_{1}$ and $R_{n}$ are closed half planes,
(c) $R_{2 k-1}$ is closed, $R_{2 k}$ is open for $k=1,2, \cdots$, and
(d) $n$ is odd.

The proof of this theorem follows easily from the above remarks.

The above remarks can be applied to describe the plane if there are $n$ fundamental regions.

For example, suppose the plane $P=R_{1} \cup R_{2} \cup R_{3}$. Since $P$ is connected, suppose $R_{1}$ and $R_{2}$ are not separated. Then either $R_{1}$ and $R_{3}$ or $R_{2}$ and $R_{3}$ are separated. Suppose $R_{2}$ and $R_{3}$ are not separated. 
By Remark 12, $R_{1}$ and $R_{3}$ are separated by $R_{2}$. Thus, by Remark 9 , $R_{1}$ and $R_{3}$ are closed and $R_{i}$ - Int $R_{i}=C_{i}$, where $C_{i}$ is a proper flowline, for $i=1,3$. Therefore, $R_{2}$ is open.

In the case $n=4,5$, or 6 , there are respectively 1,3 , or 4 possible arrangements of the fundamental regions.

3. Self-homeomorphism of the plane with no fixed points. In this section it will be shown that in certain cases, the embedding problem can be reduced to a problem of extending a continuous flow defined on an open set to the closure of the set. Sufficient conditions for this extension will be given. Some properties of the homeomorphisms on the fundamental regions will also be given.

In order to show that an open connected subset $B$ of the plane is homeomorphic to the plane, it is enough to show that every simple closed curve in $B$ separates $B$ and at least one of the components has compact closure in $B$ [12]. Thus, Theorem 5 follows.

TheOREM 5. If $R_{i}$ and $R_{i+1}$ are not separated for $i=1,2, \cdots, k-1$, then Int $\left(\cup_{j=1}^{k} R_{j}\right)$ is homeomorphic to the plane.

Proof. By the earlier remarks, we can assume that $R_{1}$ and $R_{k}$ have nonempty interior, for otherwise $R_{1}$ or $R_{k} \Phi \operatorname{Int}\left(\cup_{j=1}^{k} R_{j}\right)$. By (3), Int $R_{j}$ is connected. Since $R_{i}$ and $R_{i+1}$ are not separated, suppose for definiteness that there exists $x \in\left(R_{i}-\operatorname{Int} R_{i}\right) \cap \bar{R}_{i+1}$. By Remarks 3 and 7, there is a neighborhood $U$ of such that $U \subset R_{i-1} \cup R_{i} \cup R_{i+1}$ if $i \neq 1$ or $R_{i} \cup R_{i+1}$ if $i=1$. Therefore, $x \in \operatorname{Int}\left(\cup_{j=1}^{k} R_{j}\right)$. Thus, Int $\left(\cup_{j=1}^{k} R_{j}\right)$ is connected.

Let $B$ be a simple closed curve such that $B \subset \operatorname{Int}\left(\cup_{j=i}^{k} R_{j}\right)$. Let $x \in \operatorname{Int} B$. Since $f(x) \rightarrow \infty$ as $n \rightarrow \infty$, it follows that there is an integer $n$ such $f^{n}(x) \notin B \cup \operatorname{Int} B$. But, since $x \sim f^{n}(x)$, there is an arc $A$, where $A \subset R_{i}$ if $x \in R_{i}$ and where $A$ joins $x$ and $f^{n}(x)$. Therefore, $\{x\}$ and $A \cap B$ are in the same fundamental region. Thus, $B \cup \operatorname{Int} B \subset$ Int $\left(\cup_{j=1}^{k} R_{j}\right)$. It follows that $B \cup \operatorname{Int} B$ is compact in Int $\left(\cup_{j=1}^{k} R_{j}\right)$. Therefore, Int $\left(\cup_{j=1}^{k} R_{j}\right)$ is homeomorphic to the plane.

THEOREM 6. Suppose $R_{i}$ and $R_{i+1}$ are not separated for $i=1, \cdots$, $k-1$. Then, there is a homeomorphism $g$ : Int $\left(\cup_{i=1}^{k} R_{i}\right) \rightarrow P_{2}$, where $P_{2}$ is a plane, and $P_{2}$ under $g \mathrm{fg}^{-1}$ has at most $k$ fundamental regions.

Proof. The homeomorphism $g$ exists by Theorem 5 .

Let $x_{1}, x_{2} \in R_{j} \cap \operatorname{Int}\left(\bigcup_{i=1}^{k} R_{i}\right)$. If $R_{j} \subset \operatorname{Int}\left(\bigcup_{i=1}^{k} R_{i}\right)$, then by definition of $R_{i}$, there is a curve segment $L \subset \operatorname{Int}\left(\cup_{i=1}^{k} R_{i}\right)$ joining $x_{1}$ and $x_{2}$ for which $f^{n}(L) \rightarrow \infty$ as $\pm n \rightarrow \infty$. Since Int $R_{j} \subset \operatorname{Int}\left(\cup_{\imath=1}^{k} R_{i}\right)$, if $x_{1}, x_{2} \in$ 
Int $R_{j}$, then by (3), there is a curve segment $L \subset \operatorname{Int}\left(\cup_{i=1}^{k} R_{i}\right)$ for which $f^{n}(L) \rightarrow \infty$ as $n \rightarrow \pm \infty$. If $x_{1} \in R_{j}-$ Int $R_{j}$, then by (2), $x_{1} \in C_{1} \subset R_{j}-$ Int $R_{j}$. By Remarks 3 and $7, C_{1} \subset \operatorname{Int}\left(\cup_{i=1}^{k} R_{i}\right)$. Let $y \in R_{j}$ such that $y \notin C_{1}$. Since $x_{1} \sim y$, there is an arc $L_{1}$ joining $x_{1}$ and $y$ such that $f^{n}\left(L_{1}\right) \rightarrow \infty$ as $n \rightarrow \pm \infty$. By Remark 3, there is a subarc $L$ of $L_{1}$ such that $L$ joins $x_{1}$ to an interior point of $R_{j}$ and such that $L \subset$ $\operatorname{Int}\left(\cup_{i=1}^{k} R_{i}\right)$. Therefore, if $x_{1}, x_{2} \in R_{j} \cap \operatorname{Int}\left(\cup_{i=1}^{k} R_{i}\right)$, then there is a curve $L \subset \operatorname{Int}\left(\cup_{i=1}^{k} R_{i}\right)$ which $f^{n}(L) \rightarrow \infty$ as $n \rightarrow \pm \infty$. Let $C$ be any compact set in $P_{2}$. Then, $g^{-1}(C)$ is a compact set in $P_{1}$. Thus, there is a natural number $N$ such that if $|n|>N$, then $f^{n}(L) \cap g^{-1}(C)=\phi$. Now, $g(L)$ is a curve segment joining $g\left(x_{1}\right)$ and $g\left(x_{2}\right)$ in plane $P_{2}$. Since $f^{n}(L) \cap g^{-1}(C)=\phi, g f^{n} g^{-1} g(L) \cap C=\phi$. Therefore, $g\left(x_{1}\right)$ and $g\left(x_{2}\right)$ are in the same fundamental region.

COROLlaRy. If $k=2$, then $P_{2}$ has exactly one fundamental region under $g f g^{-1}$.

The following two embedding theorems can be considered as a continuation of the work started by Andrea [1].

THEOREM 7. If the plane under $f$ has exactly one fundamental region and if $C$ is a proper flowline, then $f$ can be embedded in a continuous flow $\Pi$, such that $\Pi(C, R)=C$.

Proof. The homeomorphism $f$ is topologically equivalent to a translation. Hence, there is a curve $K$ such that $K$ separates the plane, $f^{n}(K) \cap f^{m}(K)=\phi$ for $n \neq m$, if $z$ is a point of the plane $f^{n}(z)$ is in the strip bounded by $K$ and $f(K)$ for some unique integer $n$, and $K \cap C=\left\{x_{0}\right\}$. The plane, $P_{1}$, is homeomorphic to another plane $P_{2}$ under a homeomorphism $h$ such that $h(K)$ and $(h f) K$ are parallel straight lines and $h(C)$ from $h\left(x_{0}\right)$ to $(h f) x_{0}$ is a straight line. Now if $x \in K$ and if $0 \leqq t<1$ define

$$
\Pi_{1}(x, t)=y
$$

where $h(y)=(1-t) h(x)+t(h f) x$. If $x \in K$ and $t$ is any real number, define

$$
\Pi_{2}(x, t)=f^{n}\left(\Pi_{1}(x, s)\right),
$$

where $n+s=t, n$ is an integer, and $0 \leqq s<1$. If $x$ is in the interior of the strip bounded by $K$ and $f(K)$ or if $x \in K$, and if $t_{1}$ is any real number, define

$$
\Pi_{3}\left(x, t_{1}\right)=\Pi_{2}\left(y, t_{2}+t_{1}\right)
$$

where $h(x)=\left(1-t_{2}\right)(h f) y+t_{2} h(y)$ and $y \in K$. If $x \in P_{1}$ and $t$ is any 
real number, define

$$
\Pi(x, t)=\Pi_{3}\left(f^{-m}(x), t+m\right),
$$

where $f^{-m}(x)$ is in the strip bounded by $K$ and $f(K)$. It follows that $\Pi$ is a continuous flow and that $\Pi(x, n)=f^{n}(x)$ for every $x \in P_{1}$ and every integer $n$.

THEOREM 8. Suppose $R_{1}$ and $R_{i}$ are not separated for $i=2, \cdots$, n. Then

(a) $R_{1}$ is open

(b) $\left.f\right|_{R_{1}}$ can be embedded in a continuous flow, and

(c) if $\left.f\right|_{\bar{R}_{1}}$ can be embedded in a continuous flow $\Pi_{1}$, then $f$ can be embedded in a continuous flow $\Pi$, where $\Pi(x, r)=\Pi_{1}(x, r)$ if $(x, r) \in \bar{R}_{1}$ $X$ Reals.

(d) If $\left.f\right|_{R_{1}}$ can be embedded in a continuous flow $\Pi_{1}$, such that $\Pi_{1}$ restricted to $R_{1} \times[0,1]$ is uniformly continuous, then $f$ can be embedded in a continuous flow $\Pi$.

Proof. (a) By Remark 12, $R_{j}$ and $R_{k}$ are separated for $j \neq k$ and $j, k \neq 1$. Thus by Remark $9, R_{j}$ is closed for $j \neq 1$. Thus, $R_{1}=$ Plane - $\left(\cup_{j=2}^{n} R_{j}\right)$ is open.

(b) By Theorem 5, there is a homeomorphism $g: R_{1} \rightarrow P_{2}$ where $P_{2}$ is a plane. By Theorem $6, P_{2}$ has exactly one fundamental region under $g f g^{-1}$. Thus, $g f g^{-1}$ is topologically a translation and thus can be embedded in a continuous flow. Therefore $\left.f\right|_{R_{1}}$ can be embedded in a continuous flow.

(c) If $x \in\left(R_{2}-\right.$ Int $\left.R_{2}\right) \cap \bar{R}_{1}$ then $x \in C_{2} \subset R_{2}-$ Int $R_{2}$, where $C_{2}$ is a proper flowline. By Remarks 3 and 7, $C_{2} \subset \operatorname{Int}\left(R_{1} \cup R_{2}\right)$. It follows that Int $\left(R_{1} \cup R_{2}\right)=R_{1} \cup R_{2}$, and from the proof of Remark 9 that $R_{2}$ - Int $R_{2}=C_{2}$. From Theorem 5, $R_{1} \cup R_{2}$ and plane $P_{2}$ are homeomorphic, under a homeomorphism $g$. By the corollary to Theorem 6, $P_{2}$ has exactly one fundamental region under $g f g^{-1}$. Therefore, $g f g^{-1}$ can be embedded in a continuous flow $\sigma$ such that $\sigma(g(C), R)=g(C)$, by Theorem 7. It also can be shown that we can choose $\sigma$ such that $\Pi_{1}(x, r)=g^{-1}\left(\sigma(g(z), r)\right.$ for $g(z)=x \in C$. Now define $\Pi_{2}^{\prime}:\left(R_{1} \cup R_{2}\right) \times$ $R \rightarrow\left(R_{1} \cup R_{2}\right)$ by

$$
\Pi_{2}^{\prime}(z, r)=g^{-1}(\sigma(g(z), r))
$$

Then $\Pi_{2}^{\prime}$ is a continuous flow and $\Pi_{2}^{\prime}(z, n)=f^{n}(z)$, where $z \in R_{1} \cup R_{2}$. Define $\Pi_{2}:\left(R_{1} \cup R_{2}\right) \times R \rightarrow\left(R_{1} \cup R_{2}\right)$ by

$$
\Pi_{2}(z, r)= \begin{cases}\Pi_{2}^{\prime}(z, r) & \text { if } z \in R_{2} \\ \Pi_{1}(z, r) & \text { if } z \in \bar{R}_{1} .\end{cases}
$$


Then $\Pi_{2}$ is a continuous flow on $R_{1} \cup R_{2}$ since $\Pi_{2}^{\prime}$ and $\Pi_{1}$ agree on $C_{2}$. In the same way, define $\Pi_{i}:\left(R_{1} \cup R_{i}\right) \times R \rightarrow\left(R_{1} \cup R_{i}\right)$ for $i=2,3, \cdots$, n. Define $\Pi: P_{1} \times R \rightarrow P_{1}$ by

$$
\Pi(z, r)=\Pi_{i}(z, r) \text { if } z \in R_{i} \text { for } i=1,2, \cdots, n \text {. }
$$

The fact that $\Pi$ is a continuous flow follows from the fact that $R_{i}$ and $R_{j}$ are closed and separated for $i \neq j ; i, j \neq 1$.

(d) It is enough to show that $\left.f\right|_{\bar{R}_{1}}$ can be embedded in a continuous flow. Define $\Pi: \bar{R}_{1} \times$ Reals $\rightarrow \bar{R}_{1}$ by

$$
\Pi(x, r)=\lim _{n \rightarrow \infty} f^{k}\left(\Pi_{1}\left(x_{n}, s\right)\right),
$$

where $x_{n} \rightarrow x$ as $n \rightarrow \infty, x_{n} \in R_{1}$, and $r=k+s$, where $k$ is an integer and $0 \leqq s<1$.

Let $(x, r)$ and $\varepsilon>0$ be given. Then $r=k+s$, where $k$ is an integer and $0 \leqq s<1$. Let $x_{n} \rightarrow x$ as $n \rightarrow \infty$, where $x_{n} \in R_{1}$. By the uniform continuity of $\Pi$, on $R_{1} \times[0,1]$, there is a $\delta>0$ such that if $d\left(x_{n}, x_{m}\right)<\delta$, then $d\left(\Pi_{1}\left(x_{n}, s\right), \Pi_{1}\left(x_{m}, s\right)\right)<\varepsilon$. Since $x_{n} \rightarrow x$, there is a natural number $N$ such that if $n, m>N$, then $d\left(x_{n}, x_{m}\right)<\delta$. Therefore, $\left.\lim _{n \rightarrow \infty} f^{k} \Pi_{1}\left(x_{n}, s\right)\right)=f^{k}\left(\lim _{n \rightarrow \infty} \Pi_{1}\left(x_{n}, s\right)\right)$ exists.

Suppose $x_{n} \rightarrow x$ and $\bar{x}_{m} \rightarrow x$, where $x_{n}, \bar{x}_{m} \in R_{1}$ for every $n, m$. Let $\lim _{n \rightarrow \infty} \Pi_{1}\left(x_{n}, s\right)=A$, and $\lim _{n \rightarrow \infty} \Pi_{1}\left(\bar{x}_{m}, s\right)=B$. By the uniform continuity of $\Pi_{1}$ on $R_{1} \times[0,1]$, there is a $\delta>0$ such that if $d(y, \bar{y})<\delta$ then $d\left(\Pi_{1}(y, s), \Pi_{1}(\bar{y}, s)\right)<\varepsilon / 3$. Since $x_{n} \rightarrow x$ and $\bar{x}_{m} \rightarrow x$ as $n, m \rightarrow \infty$, there is a natural number $N$ such that if $n, m>N$, then $d\left(x_{n}, x\right)<\delta / 2$ and $d\left(\bar{x}_{m}, x\right)<\delta / 2$. Therefore $d\left(x_{n}, \bar{x}_{m}\right) \leqq d\left(x_{n}, x\right)+d\left(\bar{x}_{m}, x\right)<\delta$ if $n, m>$ $N$. Thus, $d(A, B) \leqq d\left(A, \Pi_{1}\left(x_{n}, s\right)\right)+d\left(\Pi_{1}\left(x_{n}, s\right), \Pi_{1}\left(\bar{x}_{m}, s\right)+d\left(\Pi\left(\bar{x}_{m}, s\right)\right.\right.$, $B)<\varepsilon$ for large $m$ and $n$. Since $r=k+s$, where $k$ is an integer and $0 \leqq s<1$, is a unique representation, $\Pi$ is well defined.

Since $\Pi_{1}\left(x_{n}, 0\right)=x_{n}$ if $x_{n} \in R_{1}, \Pi(x, 0)=x$ for all $x \in \bar{R}_{1}$.

Let $x_{n} \rightarrow x$ as $n \rightarrow \infty, r_{1}=k_{1}+s_{1}, r_{2}=k_{2}+s_{2}$, and $r_{1}+r_{2}=k_{3}+$ $s_{3}$, where $k_{i}$ are integers and $0 \leqq s_{i}<1$ for $i=1,2,3$, and where $x_{n} \in R_{1}$. Then, $\Pi\left(x, r_{1}+r_{2}\right)=\lim _{n \rightarrow \infty} f^{k_{3}}\left(\Pi_{1}\left(x_{n}, s_{3}\right)=\lim _{n \rightarrow \infty} f^{k_{2}}\left(\Pi_{1}\left(x_{n}\right.\right.\right.$, $\left.s_{3}+r_{1}-s_{3}+s_{2}\right)=\lim _{n \rightarrow \infty} f^{k_{2}}\left(\Pi_{1}\left(x_{n}, r_{1}+r_{2}\right)\right)=\lim _{n \rightarrow \infty} f^{k_{2}}\left(\Pi_{1}\left(x_{n}, k_{1}+s_{1}+\right.\right.$ $\left.\left.s_{2}\right)\right)=\lim _{n \rightarrow \infty} f^{k_{2}}\left(\Pi_{1}\left(\Pi_{1}\left(x_{n}, k_{1}+s_{1}\right), s_{2}\right)=\lim _{n \rightarrow \infty} f^{k_{2}}\left(\Pi_{1}\left(f^{k_{1}}\left(\Pi_{1}\left(x_{n}, s_{1}\right)\right), s_{2}\right)=\right.\right.$ $\Pi\left(\Pi\left(x, r_{1}\right), r_{2}\right)$.

Let $(x, g) \in \bar{R}_{1} \times[0,1]$. Let $\varepsilon>0$ be given. Choose a neighborhood $N \times U$ of $(x, g)$ in $\bar{R}_{1} \times[0,1]$ so that if $\left(x_{1}, g_{1}\right),\left(x_{2}, g_{2}\right) \in(N \times U) \cap\left(R_{1} \times\right.$ $[0,1])$ then $d\left(\Pi_{1}\left(x_{1}, g_{1}\right), \Pi_{1}\left(x_{2}, g_{2}\right)\right)<\varepsilon$. By the definition of $\Pi$, there exists $x_{1} \in R_{1} \cap N$ such that $d\left(\Pi_{1}\left(x_{1}, g\right), \Pi(x, g)\right)<\varepsilon$. Suppose $(\bar{x}, \bar{g}) \in$ $N \times U$. Then there exists $x_{2} \in R_{1} \cap N$ so that $d\left(\Pi_{1}\left(x_{2}, \bar{g}\right), \Pi(\bar{x}, \bar{g})\right)<\varepsilon$. Thus, $d(\Pi(x, g), \Pi(\bar{x}, \bar{g})) \leqq d\left(\Pi(x, g), \Pi_{1}\left(x_{1}, g\right)\right)+d\left(\Pi_{1}\left(x_{1}, g\right), \Pi_{1}\left(x_{1}, \bar{g}\right)\right)+$ $d\left(\Pi_{1}\left(x_{1}, \bar{g}\right), \Pi_{1}\left(x_{2}, \bar{g}\right)+d\left(\Pi_{1}\left(x_{2}, \bar{g}\right), \Pi(\bar{x}, \bar{g})\right)<\varepsilon+\varepsilon+\varepsilon=3 \varepsilon\right.$. Thus $\Pi$ is continuous on $\bar{R}_{1} \times[0,1]$. Therefore, by continuity of $f^{n}$ for every 
integer $n, \Pi$ is continuous on $\bar{R}_{1} \times$ Reals.

It follows from Theorem 8 and the remarks $\S 2$, that if the plane has either three or four fundamental regions under $f$, then the embedding problem is reduced to the problem of embedding $\left.f\right|_{R_{1}}$ in a flow that can be extended to $\bar{R}_{1}$.

REMARK 13. If $B \subset R_{k}$ is a proper flowline and $B$ separates $R_{j}$ and $R_{i}, i \neq k \neq j$, then each closed half plane defined by $B$ contains at least two fundamental regions other than $R_{k}$.

Proof. Use Remark 5 to define a homeomorphism $g$ such that $g(x)=f(x)$ if $x \in B$ and such that $g$ is topologically equivalent to a translation. Let $H^{1}$ be the half plane defined by $B$ that contains $R_{j}$, and let $H^{2}$ be the half plane defined by $B$ that contains $R_{i}$. Define $F: P_{1} \rightarrow P_{1}$, where $P_{1}$ is the plane, by

$$
F(x)= \begin{cases}g(x) & \text { if } x \in H^{1} \\ f(x) & \text { if } x \in H^{2}\end{cases}
$$

Suppose that $R_{i}$ is the only fundamental region other than $R_{k}$ that has a point in $H^{2}$. Let $z \in H^{1}-B$, and let $x \in B$. Join $z$ and $x$ by a curve $J \subset H^{2}$. Then $F^{n}(J)=g^{n}(J) \rightarrow \infty$ as $n \rightarrow \pm \infty$. Therefore, the plane has at most two fundamental regions under $F$, and thus it has exactly one. Now join $y \in R_{i}$ to $x \in B$ by a curve $K \subset H^{2}$. Then $f^{n}(K)=F^{n}(K) \rightarrow \infty$ as $n \rightarrow \pm \infty$. Thus, $x$ and $y$ are in the same fundamental region under $f$.

Corollary. If $n=3$ or 4 , then $B$ does not separate any two fundamental regions if neither contains $B$.

REMARK 14. Suppose there are exactly three fundamental regions under $f$, and $R_{i}$ and $R_{i+1}$ are not separated for $n=1,2$. If $y \in R_{2}$, $z \in R_{1}$, and $U$ is any open set such that $R_{3} \subset U$, then for every $\operatorname{arc} C$ connecting $y$ and $z$, there is an integer $N$ such that $f^{N}(C) \cap U \neq \phi$.

Proof. By Theorem 4, $R_{2}$ separates $R_{1}$ and $R_{3}$, and $R_{2}$ is open. Thus, Int $\left(R_{1} \cup R_{2}\right)=R_{1} \cup R_{2}$. By Theorem $5, R_{1} \cup R_{2}$ is homeomorphic to the plane. Suppose $g: R_{1} \cup R_{2} \rightarrow P_{2}$, where $P_{2}$ is the plane and $g$ is a homeomorphism. By Theorem $6, P_{2}$ has exactly one fundamental region under $g \mathrm{fg}^{-1}$. Suppose that for some $y \in R_{2}, z \in R_{1}$, and for some open set $U \supset R_{3}$, there is an arc $C$ connecting $y$ and $z$ such that $f^{n}(C) \cap$ $U=\phi$ for every integer $n$. Let $K$ be any compact set in $P_{1}$. Then $\left(P_{1}-U\right) \cap K$ is compact. Since $f^{n}(C) \cap U=\phi$ for every integer $n$, it follows that $f^{n}(C) \cap\left(P_{1}-U\right) \cap K=\phi$ implies $f^{n}(C) \cap K=\phi$. Now, $g f^{n} g^{-1}(g(C)) \rightarrow \infty$ as $n \rightarrow \pm \infty$. Since $\left(P_{1}-U\right) \cap K=K_{1} \subset R_{1} \cup R_{2}$, 
$g\left(K_{1}\right)$ is compact in $P_{2}$. Thus, there is a natural number $N$ such that if $|n|>N$, then $g f^{n}(C) \cap g\left(K_{1}\right)=\phi$. But that implies $f^{n}(C) \cap K_{1}=\phi$. Therefore, $y$ and $z$ are in the same fundamental region, which is a contradiction.

REMARK 15. Suppose $C_{j} \subset R_{1}$, where $C_{j}$ is a proper flowline for $j=1,2 ; H_{j}^{k}, k=1,2$, are the two closed half planes defined by $C_{j}$; and $C_{1} \subset \operatorname{Int} H_{2}^{1}$ and $C_{2} \subset \operatorname{Int} H_{1}^{2}$. Then $H_{2}^{1} \cap H_{1}^{2} \subset R_{1}$.

Proof. The proof is similar to the proof of Remark 6 .

REMARK 16. Suppose $C_{i} \subset R_{2}$, where $C_{i}$ is a proper flowline for $i=1,2$. If $C_{1}$ separates $R_{1}$ and $R_{3}$, then $C_{2}$ separates $R_{1}$ and $R_{3}$.

Proof. Suppose $C_{1}$ separates $R_{1}$ and $R_{3}$, but $C_{2}$ does not. Let $H_{i}^{k}$, $k=1,2$, be the closed half plane defined by $C_{i}$. Suppose $R_{1} \subset H_{1}^{1}$ and $R_{3} \subset H_{1}^{2}$. Since $C_{2}$ does not separate $R_{1}$ and $R_{3}$, and since each $R_{i}$ is arcwise connected, suppose $R_{1}$ and $R_{3}$ are contained in $H_{2}^{1}$. If $C_{1} \cap$ $C_{2}=\phi$, suppose $C_{2} \subset H_{1}^{2}$. Then $R_{3} \subset H_{2}^{1} \cap H_{1}^{2}$. By Remark 15, $R_{3} \subset$ $H_{2}^{1} \cap H_{1}^{2} \subset R_{2}$. If $C_{2} \subset H_{1}^{1}$, then $R_{1} \subset H_{1}^{1} \cap H_{2}^{1}$. By Remark 15, $R_{1} \subset H_{1}^{1} \cap$ $H_{2}^{1} \subset R_{2}$. If $C_{1} \cap C_{2} \neq \phi$, let $y \in C_{1} \cap C_{2}$. Then $f^{n}(y) \in C_{1} \cap C_{2}$ for every integer $n$. In this case there is a point $x \in R_{1} \cup R_{3}$, where $x$ is in the region bounded by $C_{1}$ and $C_{2}$. Since that is not possible, $C_{2}$ separates $R_{1}$ and $R_{3}$.

The following Remark easily follows from the above results.

REMARK 17. Suppose $R_{i}$ and $R_{i+1}$ are not separated for $i=1,2$. Then, there is a homeomorphism $g: \operatorname{Int}\left(\cup_{i=1}^{3} R_{i}\right) \rightarrow P_{2}$, where $P_{2}$ is a plane. If $P_{2}$ has exactly three fundamental regions under $g f g^{-1}$ if $y \in R_{2} \cap \operatorname{Int}\left(\cup_{i=1}^{3} R_{i}\right)$, if $z \in R_{1} \cap \operatorname{Int}\left(\cup_{i=1}^{3} R_{i}\right)$, and if $U$ is any open set such that $R_{3} \subset U$, then for every arc $B \subset \operatorname{Int}\left(\cup_{i=1}^{3} R_{i}\right)$ connection $y$ and $z$ there is an integer $N$ such that $f^{N}(B) \cap U \neq \phi$.

The above results gives us some information about the possible behavior of a self-homeomorphism of the plane with $n$ fundamental regions. Consider, for example, the case where the plane $P_{1}=\cup_{i=1}^{4} R_{i}$, where $R_{1}$ and $R_{i}$ are not separated for $i=2,3,4$. By Corollary to Remark 13, $R_{1}$ does not contain a proper flowline $B$ such that $B$ separates $R_{j}$ and $R_{k}$ for $j \neq k, j, k \neq 1$. By Theorem 5, Int $\left(R_{1} \cup R_{2} \cup\right.$ $\left.R_{i}\right)=R_{1} \cup R_{2} \cup R_{i}$ is homeomorphic to the plane for $i=3$, 4. By Remark 17, if $y \in R_{2}$ - Int $R_{2}$, if $U$ is any neighborhood of $y$, and if $V_{i}$ is an open set such that $V_{i} \supset R_{i}$, then there is an integer $N$ such that $f^{N}(U) \cap V_{i} \neq \phi$. 
4. Self-homeomorphisms of the plane and the closed disc with one fixed point. In this section sufficient conditions to embed selfhomeomorphisms of the plane and the closed disc will be given.

THEOREM 9. Let $f$ be an orientation preserving self-homeomorphism of the plane with one fixed point, $x_{0}$. Suppose $f^{n}(x) \rightarrow \infty$ as $n \rightarrow-\infty$ and $f^{n}(x) \rightarrow x_{0}$ as $n \rightarrow \infty$ for $x \neq x_{0}$. Then $f$ can be embedded in a continuous flow.

Proof. The proof is an immediate consequence of the theorem by Homma and Kinoshita [10] that says that such a homeomorphism is topologically equivalent to $x^{\prime}=(1 / 2) x, y^{\prime}=(1 / 2) y$.

We conclude this paper with the following embedding theorem.

THEOREM 10. Let $f$ be an orientation preserving self-homeomorphism of the closed unit disc D. Suppose

(1) if $x \in D$, then $f^{ \pm n}(x) \rightarrow x_{0}$ as $n \rightarrow \infty$, where $x_{0} \in D-\operatorname{Int} D$ and $f\left(x_{0}\right)=x_{0}$, and

(2) if $x_{1}, x_{2} \in D-\left\{x_{0}\right\}$, there is an arc $A \subset D$ such that $A$ joins $x_{1}$ and $x_{2}$ and $f^{ \pm n}(A) \rightarrow x_{0}$ as $n \rightarrow \infty$.

Then $f$ can be embedded in a continuous flow.

Proof. Let $L$ be a straight line tangent to the circle $C=D-$ Int $D$ at $x_{0}$. Let $L_{1}$ be the ray along $L$ from $x_{0}$. The subspace $P_{1}$ $L_{1}$ is homeomorphic to a plane $P_{2}$. Suppose $g$ is a homeomorphism of $P_{1}-L_{1}$ onto $P_{2}$ sending $C-\left\{x_{0}\right\}$ onto the $y$-axis of plane $P_{2}$. If $x \in \operatorname{Int} D$, let $H_{1}$ be the closed half plane defined by the $y$-axis of plane $P_{2}$ containing $g(x)$. It follows that $g^{-1}\left(H_{1}\right)=D-\left\{x_{0}\right\}$. Now define $F$ : $P_{2} \rightarrow P_{2}$ by

$$
\begin{aligned}
F(x, y) & =\left(x,\left(g f g^{-1}(0, y)_{y}\right)\right. & & \text { if }(x, y) \in P_{2}-H_{1} \\
& =g f g^{-1}(x, y) & & \text { if }(x, y) \in H_{1} .
\end{aligned}
$$

$F$ is orientation preserving self-homeomorphism of plane $P_{2}$ with no fixed points. If $z \in P_{2}-H_{1}$, then clearly $z$ is in the same fundamental region as the $y$-axis of $P_{2}$. Let $x_{1}, x_{2} \in H_{1}$ and let $K$ be any compact set in $P_{2}$. Since $g^{-1}(K)$ is a compact set in $P_{1}$ and $x_{0} \notin g^{-1}(K)$, it follows that there is an arc $A$ joining $g^{-1}\left(x_{1}\right)$ and $g^{-1}\left(x_{2}\right)$ such that $f^{ \pm n}(A) \cap$ $g^{-1}(K)=\phi$ if $n>N$ for some $N$. But that implies $F^{ \pm n}(g(A)) \cap K=$ $\phi$ if $n>N$. Thus $P_{2}$ has exactly one fundamental region under $F$, and consequently can be embedded in a flow $\Pi$ which leaves the $y$-axis of $P_{2}$ invariant by Theorem 7. It follows that $\Pi\left(H_{1}, r\right)=H$ for every real number $r$. Thus, $f$ is restricted to $D-\left\{x_{0}\right\}$ can be embedded in a flow $\sigma_{1}$. Now define $\sigma: D \times R \rightarrow D$ by 


$$
\begin{array}{ll}
\sigma(d, r)=\sigma_{1}(d, r) & \text { if } d \in D-\left\{x_{0}\right\}, r \in R, \\
\sigma\left(x_{0}, r\right)=x_{0} & \text { if } r \in R .
\end{array}
$$

$\sigma$ is a continuous flow if it is continuous at $x_{0}$. Let $U$ be an open neighborhood of $x_{0}$ in $D$. Then $D-U$ is compact in $P_{1}$. Thus $g(D-U)$ is compact in $P_{2}$. There exists a compact set $K \subset P_{2}$ such that if $x \in P_{2}-K$ and if $\left|t-t_{0}\right|<1$ then $\Pi(x, t) \in P_{2}-g(D-U)$. From that it follows that if $x \in\left[g^{-1}\left(P_{2}\right)-g^{-1}(K)\right] \cap D$ and if $\left|r-r_{0}\right|<1$, then $\sigma(x, r) \in U$. Thus, $\sigma$ is continuous at $\left(x, r_{0}\right)$.

\section{REFERENCES}

1. S. A. Andrea, On homeomorphisms of the plane which have no fixed points, Abhandlungen aus dem Mathematische Seminar der Universitat Hamburg, 30 (1967), 61-74.

2. N. J. Fine and G. E. Schweigert, On the group of homeomorphisms of an arc, Annals of Math. 62 (1955), 237-253.

3. N. E. Foland, An embedding theorem for discrete flows on a closed 2-cell, Duke Math. J. 33 (1966), 441-444.

4. - An embedding theorem for contracting homeomorphisms, Mathematical Systems Theory, vol. 3, number 2 (1969), 166-169.

5. N. E. Foland and W. R. Utz, The Embedding of Discrete Flows in Continuous Flows, Ergodic Theory, Academic Press: New York, 1963.

6. M. K. Fort, Jr., The embedding of homeomorphisms in flows, Proc. Amer. Math. Soc. 6 (1955), 960-967.

7. W. H. Gottschalk, Minimal sets: an introduction to topological dynamics, Bull. Amer. Math. Soc., 64 (1958), 336-351.

8. W. H. Gottschalk and G. A. Hedlund, Topological Dynamics, Amer. Math. Soc. Colloq. Publ., New York, 1955.

9. D. W. Hall and G. L. Spencer, II, Elementary Topology, John Wiley and Sons, Inc.: New York, 1955.

10. Tatsuo Homma and Shin'ichi Kinoshita, On the regularity of homeomorphisms of $E^{n}$, J. Math. Soc. of Japan, 5 (1953), 365-371.

11. W. R. Utz, The embedding of a linear discrete flow in a continuous flow, Colloquium Mathematicum, 15 (1966), 263-270.

12. E. R. Van Kampen, On some characterizations of 2-dimensional manifolds, Duke Math. J., 1 (1935), 74-93.

Received October 24, 1969 and in revised form October 19, 1971. This work is part of the author's University of Missouri doctoral dissertation under the direction of Professor W. R. Utz.

The author wishes to acknowledge the referee's many helpful comments. 


\section{PACIFIC JOURNAL OF MATHEMATICS}

\section{EDITORS}

\author{
H. SAMELSON \\ Stanford University \\ Stanford, California 94305

\section{R. HoвBY} \\ University of Washington \\ Seattle, Washington 98105
}

\section{J. DugundJI}

Department of Mathematics University of Southern California Los Angeles, California 90007

\author{
RichaRd ARENS \\ University of California \\ Los Angeles, California 90024
}

\section{ASSOCIATE EDITORS}
E. F. BECKENBACH
B. H. NEUMANN
F. WOLF
K. YoshidA

\section{SUPPORTING INSTITUTIONS}

\author{
UNIVERSITY OF BRITISH COLUMBIA \\ CALIFORNIA INSTITUTE OF TECHNOLOGY \\ UNIVERSITY OF CALIFORNIA \\ MONTANA STATE UNIVERSITY \\ UNIVERSITY OF NEVADA \\ NEW MEXICO STATE UNIVERSITY \\ OREGON STATE UNIVERSITY \\ UNIVERSITY OF OREGON \\ OSAKA UNIVERSITY
}

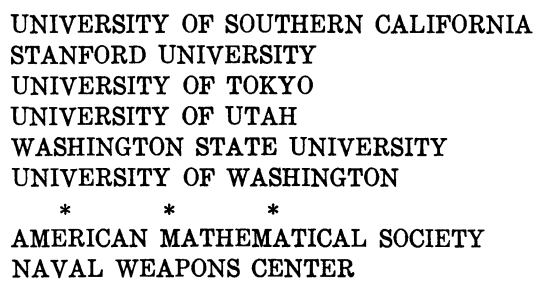

The Supporting Institutions listed above contribute to the cost of publication of this Journal, but they are not owners or publishers and have no responsibility for its content or policies.

Mathematical papers intended for publication in the Pacific Journal of Mathematics should be in typed form or offset-reproduced, (not dittoed), double spaced with large margins. Underline Greek letters in red, German in green, and script in blue. The first paragraph or two must be capable of being used separately as a synopsis of the entire paper. The editorial "we" must not be used in the synopsis, and items of the bibliography should not be cited there unless absolutely necessary, in which case they must be identified by author and Journal, rather than by item number. Manuscripts, in dup icate if possible, may be sent to any one of the four editors. Please classify according to the scheme of Math. Rev. Index to Vol. 39. All other communications to the editors should be addressed to the managing editor, Richard Arens, University of California, Los Angeles, California, 90024.

50 reprints are provided free for each article; additional copies may be obtained at cost in multiples of 50 .

The Pacific Journal of Mathematics is published monthly. Effective with Volume 16 the price per volume (3 numbers) is $\$ 8.00$; single issues, $\$ 3.00$. Special price for current issues to individual faculty members of supporting institutions and to individual members of the American Mathematical Society: $\$ 4.00$ per volume; single issues $\$ 1.50$. Back numbers are available.

Subscriptions, orders for back numbers, and changes of address should be sent to Pacific Journal of Mathematics, 103 Highland Boulevard, Berkeley, California, 94708.

PUBLISHED BY PACIFIC JOURNAL OF MATHEMATICS, A NON-PROFIT CORPORATION

Printed at Kokusai Bunken Insatsusha (International Academic Printing Co., Ltd.), 270, 3-chome Totsuka-cho, Shinjuku-ku, Tokyo 160, Japan. 


\section{Pacific Journal of Mathematics}

\section{Vol. 41, No. 2 December, 1972}

Tom M. (Mike) Apostol, Arithmetical properties of generalized Ramanujan sums .......................................... 281

David Lee Armacost and William Louis Armacost, On p-thetic groups ........ 295

Janet E. Mills, Regular semigroups which are extensions of groups .......... 303

Gregory Frank Bachelis, Homomorphisms of Banach algebras with minimal ideals ................................................ 307

John Allen Beachy, A generalization of injectivity .................. 313

David Geoffrey Cantor, On arithmetic properties of the Taylor series of rational functions. II.........................................

Václáv Chvátal and Frank Harary, Generalized Ramsey theory for graphs. III.

Small off-diagonal numbers .................................. 335

Frank Rimi DeMeyer, Irreducible characters and solvability of finite groups . . . . 347

Robert P. Dickinson, On right zero unions of commutative semigroups........ 355

John Dustin Donald, Non-openness and non-equidimensionality in algebraic

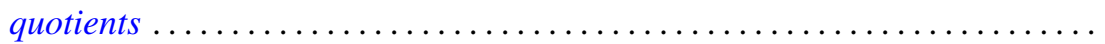

John D. Donaldson and Qazi Ibadur Rahman, Inequalities for polynomials with a prescribed zero ........................................ 375

Robert E. Hall, The translational hull of an $N$-semigroup ................ 379

John P. Holmes, Differentiable power-associative groupoids.............. 391

Steven Kenyon Ingram, Continuous dependence on parameters and boundary data for nonlinear two-point boundary value problems .

Robert Clarke James, Super-reflexive spaces with bases ..........

Gary Douglas Jones, The embedding of homeomorphisms of the plane in

continuous flows...............................

Mary Joel Jordan, Period $H$-semigroups and $t$-semisimple periodic

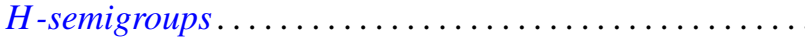

Ronald Allen Knight, Dynamical systems of characteristic 0

Kwangil Koh, On a representation of a strongly harmonic ring by sheaves...

Hui-Hsiung Kuo, Stochastic integrals in abstract Wiener space. ..

Thomas Graham McLaughlin, Supersimple sets and the problem of extending a

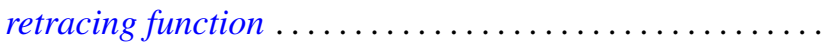

William Nathan, Open mappings on 2-manifolds .

M. J. O'Malley, Isomorphic power series rings

Sean B. O'Reilly, Completely adequate neighborhood systems and metrization

Qazi Ibadur Rahman, On the zeros of a polynomial and its derivative...

Russell Daniel Rupp, Jr., The Weierstrass excess function ..

Hugo Teufel, A note on second order differential inequalities and functional

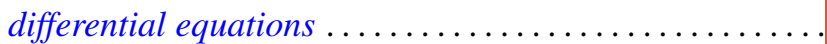

M. J. Wicks, A general solution of binary homogeneous equations over free 